\title{
Linx
}

Revue des linguistes de l'université Paris X Nanterre

$55 \mid 2006$

Thème et thématisation

\section{La structure de l'information dans les questions : quelques remarques sur la diversité des formes interrogatives en français}

\section{Claire Beyssade}

\section{OpenEdition}

\section{Journals}

Édition électronique

URL : http://journals.openedition.org/linx/470

DOI : $10.4000 / \operatorname{lin} x .470$

ISSN : 2118-9692

Éditeur

Presses universitaires de Paris Nanterre

\section{Édition imprimée}

Date de publication : 1 décembre 2006

Pagination : 173-193

ISSN : 0246-8743

\section{Référence électronique}

Claire Beyssade, "La structure de l'information dans les questions : quelques remarques sur la diversité des formes interrogatives en français », Linx [En ligne], 55 | 2006, mis en ligne le 24 février 2011, consulté le 19 avril 2019. URL : http://journals.openedition.org/linx/470 ; DOI : 10.4000/linx.470 


\title{
La structure de l'information dans les questions : quelques remarques sur la diversité des formes interrogatives en français
}

\author{
Claire Beyssade \\ CNRS-Institut Jean Nicod - UMR 8129
}

\begin{abstract}
Depuis une vingtaine d'années, beaucoup de travaux en linguistique se sont intéressés à la structure de l'information. On a observé que des énoncés qui ont le même contenu propositionnel (i.e. qui ont les mêmes conditions de vérité) peuvent présenter des formes distinctes, la variation pouvant porter sur l'ordre des mots, sur la réalisation prosodique ou encore sur l'emploi de certaines constructions grammaticales comme la topicalisation ou le clivage. Ce serait une des tâches de la grammaire que de rendre compte de ce fait.

Cependant, la plupart de ces travaux ont porté uniquement sur la structure de l'information des phrases assertives, très peu ont pris en considération les autres types de phrases (interrogatives, jussives ou exclamatives). Dans cet article, nous allons nous interroger sur la structure de l'information dans les questions en français ${ }^{1}$. Le français est une langue dans laquelle les questions peuvent prendre des formes très diverses (mot interrogatif in situ, mot interrogatif antéposé (en tête de phrase), question avec ou sans inversion clitique, question déclarative...), il est donc légitime de se demander si

${ }^{1}$ Je veux remercier ici les chercheurs avec lesquels j'ai travaillé sur ce sujet, tout particulièrement J.-M. Marandin et le groupe de travail sur le sens de l'intonation (E. Delais, M. de Fornel, C. Portes et A. Rialland) ainsi que les membres de l'ACI dirigée par C. Jakubowicz. Ces réflexions font suite à des séances de travail où nous avons étudié à la fois des exemples construits et des exemples attestés, extraits en particulier du corpus Ester.
\end{abstract}


Claire Beyssade

le choix de telle ou telle forme interrogative est libre ou contraint, et dans le cas où le choix est contraint, si ces contraintes sont de nature syntaxique, sémantique, ou discursive.

Nous commencerons par un bref rappel des travaux sur la structure de l'information, puis nous passerons à l'étude plus spécifique des questions : après avoir considéré la multiplicité des formes interrogatives disponibles en français, nous en viendrons à l'étude du sens des questions, et nous verrons comment la sémantique structurée capte des différences qui échappent aux autres cadres d'analyse. Enfin, nous nous attacherons à l'étude de certains contrastes, notamment au contraste entre questions biaisées et non biaisées, entre questions in situ et questions antéposées, et nous terminerons par quelques considérations sur le rôle de l'accentuation dans les questions.

\section{La structure de l'information}

Sans remonter aux origines qui datent probablement du dix-neuvième siècle avec les écrits de Paul, on peut dire que les travaux sur la structure informationnelle ont une longue histoire, qui passe en particulier par Halliday (1967), Jackendoff (1972), Lambrecht (1994), Engdahl et Vallduví (1994) et plus récemment par les travaux menés sur les grammaires de conversation (Engdahl (2006), Ginzburg (à par.)). Nous n'allons pas ici présenter le détail de ces travaux, mais juste revenir sur les concepts et la terminologie qui ont été mis en place et qui permettent de décrire les phénomènes.

\subsection{Faits de base}

La structure de l'information concerne la relation entre ce qui est dit et la manière dont cela est dit, entre ce qui est dit et les moyens syntaxiques ou prosodiques utilisés pour le dire. Le fait de base à l'origine des travaux sur la structure informationnelle concerne la position de l'accent nucléaire en anglais, noté ici par des majuscules. En (1), l'accent nucléaire de l'énoncé est porté par le constituant qui résout la question, ou bien par le dernier item lexical quand la question est générale, du type "Que se passe-t-il ?", comme en (1-iv).

(1) i A: Who did Paul introduce to Sue ?

Qui Paul a-t-il présenté à Sue?

B: a. Paul introduced BILL to Sue.

b. \# Paul introduced Bill to SUE.

Paul a présenté Bill à Sue

ii A: Who did Paul introduce Bill to?

A qui Paul a-t-il présenté Bill ?

B: a. Paul introduced Bill to SUE.

b. \# Paul introduced BILL to Sue.

Paul a présenté Bill à Sue 
iii A: What did Paul do at the party?

Qu'a fait Paul à la soirée?

B: a. Paul introduced Bill to SUE.

b. \# Paul introduced BILL to Sue.

Paul a présenté Bill à Sue

iv. A: What happened at the party?

Que s'est-il passé à la soirée?

B: a. Paul introduced Bill to SUE.

b. \# Paul introduced BILL to Sue.

Paul a présenté Bill à Sue

Les contrastes illustrés dans les réponses montrent que la position de l'accent est contrainte, le dièse indique que la réponse, bien que bien formée syntaxiquement, est inappropriée en contexte.

On a des données comparables en français, même si le contraste est sans doute moins connu et peut-être moins audible, car le français a une prosodie très différente de l'anglais. Notre but n'étant pas ici d'écrire une grammaire de l'intonation du français (cf. Di Cristo (1999), Delais-Roussarie (2005) entre autres), nous ne nous prononcerons pas sur la nature phonologique des tons hauts $(\mathrm{H})$ et bas (B) dans les exemples suivants (pitchs, tons de frontières...). Il nous suffit de constater qu'il existe bien un contraste en français, analogue au contraste (1) (cf. Beyssade \& al. (2003), Beyssade \& al. (2004)).

(2) i A: Qui joue de la mandoline?

B: a. Jean-Marie (B) joue de la mandoline (B).

b. \# Jean-Marie joue de la mandoline (B).

ii A: Qu'est-ce qui se passe?

B: a. \# Jean-Marie (B) joue de la mandoline (B).

b. Jean-Marie joue de la mandoline (B).

S'ajoute à cela l'analyse des réponses dites elliptiques : sont-elles premières, ou résultent-elles d'un effacement, que peut-on effacer, quand...?

(1') A: Who did Paul introduce to Sue ?

B: a. BILL.

b. \# BILL to Sue.

(2') A: Qui joue de la mandoline?

B: Jean-Marie.

L'information peut aussi être structurée au travers de l'ordre des mots, ou de l'emploi de constructions spécifiques. Les phrases suivantes, qui ont le même contenu propositionnel, ne peuvent pas indifféremment apparaittre dans tous les contextes. Le contraste donné en (3') illustre l'impact des constructions sur la structure de l'information. 

a. Jean a fait le gâteau.
b. Jean, il a fait le gâteau.
b'. Le gâteau, Jean l'a fait.
c. C'est le gâteau que Jean a fait.
c'. C'est Jean qui a fait le gâteau.

(3') A: Qui a fait le gâteau ?

B: a. Jean.

a'. Jean a fait le gâteau.

b. ? Jean, il a fait le gâteau.

b'. Le gâteau, Jean l'a fait.

c. C'est Jean qui a fait le gâteau.

c'. \# C'est le gâteau que Jean a fait.

(4) illustre la relation qui existe en Italien entre position du sujet et structure de l'information : le groupe nominal sujet doit être postverbal quand il résout une question; il doit être préverbal quand le sujet ne constitue pas à lui seul la réponse à la question, mais est un des constituants de la réponse (cf. Marandin (2003)).

(4) i. A.: Chi ha rotto il vaso ?

Qui a cassé le vase?

B.: i. L'ha rotto Maria

ii. \# Maria l'ha rotto Maria l'a cassé

ii. A. : Cos'è successo al vaso? Que s'est-il passé avec le vase?

B.: i. \# L'ha rotto Maria

ii. Maria l'ha rotto

Maria l'a cassé

\subsection{Analyses}

On analyse en général ces exemples en distinguant dans un énoncé deux parties, le fond et le focus. Le fond correspond à l'information «ancrée » dans le contexte, alors que le focus correspond à l'information nouvelle, apportée par la réponse à la question. Les termes employés pour dénommer ces deux parties varient avec les auteurs.

Jackendoff (1972) conserve le terme de focus, mais l'oppose à la présupposition.

Lambrecht (1994) lui utilise le terme de focus, mais distingue trois cas, selon la nature syntaxique de l'élément focalisé. Si c'est la phrase tout entière, comme dans (1iv), il parle de phrase all-focus. S'il s'agit du groupe verbal, comme dans (1-iii), il parle de predicate focus, et s'il s'agit d'un argument de la phrase, comme en (1-i) et en (1-ii), il parle d'argument focus. Dans les énoncés de type predicate focus, les dimensions syntaxique, prédicative et informationnelle coïncident, alors que dans les énoncés de type argument focus, les partitions syntaxique, prédicative et informationnelle ne sont pas les mêmes. 
Quant à Vallduví (1992), il propose une variante de l'architecture de Jackendoff. Son idée est de partitionner le fond (c'est-à-dire la partie présupposée) en deux parties, qu'il appelle Link et Tail. Le Link correspond à ce dont on parle, que d'autres appellent le topic. C'est un objet, une entité, qui peut être vue comme une adresse, un pointeur vers l'endroit où enregistrer l'information nouvelle ou focalisée. En ( 3 'b') par exemple, le gâteau est le Link : l'information que Jean a fait le gâteau vient s'ajouter à ce qu'on sait du gâteau. Vallduvi appelle Tail le reste du fond, le fond privé du Link.

Enfin, l'analyse de Steedman (2000) peut être vue comme une traduction formelle dans le cadre de la grammaire catégorielle des idées de Hallyday. Il y a un premier niveau de partition du contenu en Thème - Rhème (le Thème étant vu comme une propriété, il est comparable à la présupposition de Jackendoff), puis un second niveau de partition, le Thème comme le Rhème étant chacun divisé en Focus et Background. C'est la localisation des accents nucléaires qui justifie cette seconde partition, elle permet de distinguer les constituants importants du Thème ou du Rhème.

Formellement donc, le Thème (de Halliday et Steedman), la présupposition (de Jackendoff) et le fond (de Vallduvî) s'équivalent.

\subsection{Terminologie}

Même si, comme on vient de le voir, la terminologie diffère d'un auteur à l'autre, en fait, les phénomènes qu'ils cherchent à capter sont bien les mêmes. Pour éviter toute ambiguiité, nous allons préciser le cadre dans lequel nous situons notre analyse.

Pour commencer, on soulignera un fait crucial : dans tous les exemples mentionnés avant, la partition du contenu s'est faite en référence à la paire question-réponse. C'est que les énoncés sont vus comme le vecteur d'un transfert d'information entre les interlocuteurs. Si l'on cherche à modéliser ce transfert pour rendre compte des contraintes qui pèsent sur l'emploi en discours de telle ou telle forme, il nous faut un modèle de la conversation et des agents conversationnels. Nous allons présenter ici les grandes lignes de ce modèle, qui pour une large part, s'inspire des travaux sur les grammaires de conversation de Engdahl \& Vallduví (1994) et Ginzburg (à par.).

Chaque énoncé est associé à un contenu informationnel, et le discours, qu'il soit dialogique ou monologique, est vu comme un échange d'information ou de contenu entre des interlocuteurs. On associe à chaque participant à la conversation un état d'information, comprenant, entre autres choses :

(i) un ensemble de propositions, noté FACTS, qui représente les croyances et connaissances de cet agent, et

(ii) un ensemble ordonné de questions, noté QUD, qui représente l'ensemble des questions que l'agent cherche à résoudre au cours la conversation.

La question qui se trouve au haut de cet ensemble ordonné est appelée la question maximale (Qmax), c'est celle que le locuteur cherche à résoudre. Les autres questions dans QUD correspondent aux questions que le locuteur se pose. Il peut s'agir de sous-questions de Qmax, ou de questions en rapport avec Qmax, ou encore d'autres questions, posées précédemment dans le discours mais laissées en attente d'une résolution ultérieure. 
Claire Beyssade

On analyse le sens d'un énoncé au travers de l'impact qu'il a sur ces deux ensembles, qu'on notera FACTS et QUD. Quand une question est posée, elle vient s'ajouter à QUD et devient la question maximale. Quand une question est résolue, elle est enlevée de QUD.

Quand une assertion est proférée, une mise à jour est effectuée dans FACTS. Mais, comme l'a noté Ginzburg (1997), il ne suffit pas qu'un locuteur asserte p pour que p devienne une croyance partagée par l'ensemble des interlocuteurs. Une assertion réussit uniquement lorsqu'elle n'est pas rejetée ni réfutée. Si toute réfutation doit être explicite dans le dialogue, en revanche, l'acceptation peut rester tacite. Quand elle est explicite, on parle d'acknowlegment.

(5) A: Jean est venu hier.

B: a. Non, il est venu avant hier. Rejet

b. Ah bon. / Humhum. Acknowledgment

c. Et j'ai enfin pu lui parler. acceptation implicite

Pour rendre compte du fait qu'une assertion peut être rejetée, Ginzburg a proposé de considérer que l'impact d'une assertion sur la conversation était double. Une assertion conduit à mettre à jour, non seulement FACTS mais aussi QUD. L'assertion de p conduit à ajouter p dans FACTS, et à ajouter dans QUD la question polaire construite à partir de $\mathrm{p}$, comme illustré en (6). On suppose que A commence la conversation et donc que QUD est vide.

(6) A: Jean est absent

Mise à jour de FACTS : FACTS $\cup$ absent(j)

Mise à jour de QUD : QUD $=<$ ? absent (j) $>$

B: a. Oui.

Mise à jour de FACTS : pas de changement

Mise à jour de QUD : QUD $=<>$

b. Tu crois?

Mise à jour de FACTS : pas de changement

Mise à jour de QUD : QUD $=<$ ? $\operatorname{croit}(\mathrm{A}, \operatorname{absent}(\mathrm{j}))>$

On voit donc que QUD est mis à jour aussi bien quand le locuteur fait une assertion que lorsqu'il pose une question. C'est donc le lieu de modélisation de l'interaction dans le dialogue. A première vue, cela peut sembler étrange, mais en fait, isoler QUD permet de ne pas préjuger qu'une assertion sera acceptée, et donc offre un modèle qui permet de rendre compte aussi bien de l'acceptation que du refus d'une assertion. Par ailleurs, QUD sert explicitement chez Ginzburg et Engdahl à modéliser l'articulation entre fond et focus d'un énoncé. Toute assertion étant replongée dans le contexte d'une question. On dira que l'assertion est appropriée si et seulement si le fond de l'assertion correspond exactement à la question posée. S'il existe un décalage entre QUD et le fond, alors l'assertion est, en règle générale, inappropriée. 
Enfin, en plus des notions de fond et de focus, on utilisera la notion de thème de discours, reprise à Büring (1997). Le thème de discours joue un rôle essentiel dans l'analyse des contrastes discursifs, comme illustré dans les exemples qui suivent.

(7) A: Que deviennent les parents de Jean?

B: a. Ils sont à la retraite.

b. Sa mère est toujours professeur au collège.

(8) A: As-tu envoyé les invitations pour le vernissage ?

B: a. Oui.

b. Quelle importance, puisque la Poste est en grève.

On peut dire qu'en (7) et (8), la réponse donnée par B en (a) continue le thème de discours, alors qu'en (b), elle déplace la question : B fournit une réponse partielle ou en rapport avec la question, mais pas une réponse directe ni complète à la question. $B$ change partiellement le thème de discours en (7), en passant des parents de Jean à la mère de Jean, et il le change de façon plus radicale en (8).

Nous faisons donc ici un usage sémantique ou sémantico-pragmatique des concepts de fond, focus et thème de discours. On ne définit pas le focus ou le thème de discours par le fait qu'il porte telle ou telle marque prosodique, mais par le rôle qu'il joue dans l'organisation du contenu de l'information véhiculée par l'énoncé.

\subsection{Quid des questions?}

On voit donc l'importance des questions dans les recherches sur la structure de l'information. C'est la présence d'une question dans le contexte qui permet de déterminer l'élément focalisé dans l'assertion. Toute assertion est en fait replacée dans le contexte d'une question, que cette question reste implicite, ou soit explicite. Mais si les questions jouent un rôle crucial dans les travaux sur la structure de l'information, c'est en tant qu'outil, pour déterminer la partition du contenu dans les assertions. L'analyse des questions en tant que telles a en général été oubliée ${ }^{2}$. On s'est souvent contenté de dire que dans le cas des questions partielles, avec mots interrogatifs, c'est le mot interrogatif lui-même qui était focalisé. Cette stipulation s'appuyait sur plusieurs observations, au nombre desquelles le fait que dans une question, le mot interrogatif ne peut jamais être élidé, et le fait aussi qu'en anglais, il est accentué. Il semble néanmoins qu'il faille aller beaucoup plus loin dans l'analyse des questions, surtout si l'on s'intéresse à tous les types de textes, et pas seulement aux discours monologiques. On doit par exemple se demander si la distinction entre phrase all focus et phrase argument focus s'applique aux interrogatives. Il n'y a sans doute pas beaucoup de sens à dire qu'en (9) a et b, c'est le clitique inversé ou est-ce que qui est focalisé.

$$
\begin{aligned}
& \text { a. Jean est-il venu? } \\
& \text { b. Est-ce que Jean est venu ? }
\end{aligned}
$$

\footnotetext{
${ }^{2}$ A l'exception de quelques travaux, comme en particulier Lambrecht et Michaelis (1998).
} 
Claire Beyssade

Dès lors qu'on élargit le champ d'investigation aux phrases non assertives, on voit qu'il devient impossible de faire fonctionner le modèle de la paire question réponse pour analyser la structure de l'information des phrases. On voit aussi qu'on ne peut plus identifier le focus et l'information nouvelle : si les mots interrogatifs sont focalisés dans les phrases interrogatives, quel sens y a-t-il à dire qu'ils apportent une information nouvelle? En quoi qui en (3') apporte-t-il une information nouvelle ? Au mieux, c'est la réponse à la question qui apportera une information nouvelle, la question, elle, oriente seulement le débat. Avant de répondre à ces questions, nous allons d'abord décrire la grande variété de formes disponibles en français pour questionner, et montrer comment analyser les sens de ces formes dans le cadre formalisé de la sémantique structurée.

\section{Les questions}

\subsection{La multiplicité des formes "interrogatives" en français}

Pour poser une question en français, un locuteur a à sa disposition une multiplicité de formes possibles. On en donne en (10) une liste ${ }^{3}$. C'est une spécificité du français que d'offrir une telle variété de procédures de formation des questions.

$$
\begin{aligned}
& \text { a. Qui (diable) Jean a-t-il invité ? } \\
& \text { b. Qui (diable) Jean a invité ? } \\
& \text { c. Qui est-ce que Jean a invité ? } \\
& \text { d. C'est qui que Jean a invité ? } \\
& \text { e. Jean a invité qui ? }
\end{aligned}
$$

Les formes (a)-(c) sont clairement interrogatives, elles sont marquées syntaxiquement par l'emploi d'un mot interrogatif (qui) en tête de phrase, à quoi s'ajoute l'inversion clitique en a, ou le renforcement par est-ce que en c. Dans les trois cas, on parlera d'une question avec mot interrogatif antéposé, ce mot n'occupant pas en surface la position habituellement assignée aux groupes nominaux ayant la même fonction. En grammaire générative, on parle d'un déplacement ou d'un mouvement d'une position en structure profonde, vers une position de surface qui se trouve dans la périphérie gauche de la phrase.

La simple description des formes d et e pose plus de problème. En d, on a une phrase clivée construite avec un mot interrogatif, et en e, le mot interrogatif n'a pas

\footnotetext{
${ }^{3}$ Nous mentionnons dans ces exemples la possibilité d'employer diable dans les tours interrogatifs. Faute de place, nous ne pouvons pas discuter le statut précis de ce terme dans les constructions interrogatives (cf. Obenauer (1994)). Disons seulement qu'il faut bien distinguer le diable des tours interrogatifs du diable exclamatif, qu'on trouve en emploi isolé, ou précédé de que:

(i) Diable! C'est un peu cher.

(ii) Un peu de dignité, que diable!

Par ailleurs, si nous avons mentionné diable, et pas d'autres expressions pouvant renforcer l'interrogation comme donc par exemple, c'est parce que diable à la différence de donc, n'a pas les propriétés syntaxiques d'un adverbe mais est beaucoup plus contraint que ce dernier. Sur la valeur de donc dans les questions, voir Culioli (1990).
} 
bougé. On parle souvent de question in situ dans ce genre de cas. Le problème qui reste ouvert concerne le type syntaxique de ces phrases. Il est courant de considérer qu'on a ici des phrases interrogatives, parce qu'on a un mot interrogatif. Mais dans la mesure où rien dans l'ordre des mots ou dans la hiérarchie des constituants ne distingue ces phrases de déclaratives questionnantes du type de (11), souvent associées à une intonation montante, on peut se demander s'il est encore justifié d'employer le terme d'interrogative pour décrire (10) d et e. Pour notre part, nous ne faisons pas ce choix, et parlons de questions déclaratives clivées ou in situ, et non pas de phrases interrogatives. Nous réservons le terme d'interrogative à la syntaxe, quand on peut constater un changement dans l'ordre des mots (inversion sujet verbe ou redoublement clitique) ou dans les relations de dépendance (comme la présence d'un élément dans la position de complémenteur) et nous parlons de question pour faire référence à l'usage qui est fait des énoncés. Nous analysons les mots interrogatifs in situ comme des indéfinis, reprenant une idée mise en avant par Haspelmath (1997), qui, dans ses travaux sur les pronoms indéfinis, a montré que dans de nombreuses langues on trouve des interrogatifs nus employés comme des indéfinis.

$$
\begin{aligned}
& \text { a. C'est Catherine que Jean a invitée? } \\
& \text { b. Jean a invité Catherine? }
\end{aligned}
$$

On a observé que la gamme de formes possibles décrites en (10) n'est pas toujours disponible. Ainsi par exemple, tous les mots interrogatifs ne peuvent pas rester in situ. (12) montre un contraste entre les mots interrogatifs comme qui, où, ou comment d'une part et pourquoi ou que d'autre part, qui ne se trouvent jamais in situ. On explique (12b) en analysant que comme un clitique, une forme faible, dont la forme forte correspondante serait quoi. On observe en effet une distribution complémentaire entre que et quoi (cf. 13). Mais aucune explication de ce type ne convient pour pourquoi.

a. ??? Jean saigne pourquoi ?4

b. *Marie a acheté que ?

a. Marie a acheté quoi /* que ?

b. Que / *Quoi Marie a-t-elle acheté ?

Les formes avec diable, elles non plus, ne peuvent jamais être in situ (Obenauer (1994)).

$$
\begin{aligned}
& \text { a. * Jean a rencontré qui diable ? } \\
& \text { b. * Il est parti où diable? }
\end{aligned}
$$

\footnotetext{
Bellunese.
(i) A: - Je viendrai avec Jean.
B: - Tu viendras avec qui?
(ii) Est-il bean (?/!)

${ }^{4}$ Exemple dû à Obenauer, qui le juge agrammatical, et le fait précéder d'une étoile. Dans cet article, on laisse de côté les questions écho, comme (i), ainsi que les interprétations que je qualifierai d'exclamatives des phrases interrogatives, comme en (ii). Certains de ces emplois non standard ont été étudiés en particulier par Obenauer (2005) : les questions de surprise - désapprobation en 
Claire Beyssade

Chang (1997) et Cheng \& Rooryck (2000) ont observé d'autres restrictions sur les questions in situ. Ils soutiennent que, en dehors des cas de questions échos, ni une expression quantifiée, ni une négation, ni un modal ne peut précéder un mot interrogatif, d'où le contraste entre (15) et (16).

a. \# Tous les étudiants ont rencontré qui ?

b. \# Chaque étudiant a rencontré qui ?

c. \# Jean n’a pas rencontré qui ?

d. \# Jean peut rencontrer qui ?

e. \# Jean admire toujours qui ?

(16) a. Qui tous les étudiants ont-ils rencontré ?

b. Qui chaque étudiant a-t-il rencontré ?

c. Qui Jean n'a-t-il pas rencontré ?

d. Qui Jean peut-il rencontrer?

e. Qui Jean admire-t-il toujours?

A regarder de près, ces données semblent discutables ${ }^{5}$. Il faut certainement moduler les jugements. Les phrases (15) semblent étranges hors contexte, mais replacées en contexte, elles seraient tout à fait acceptables. Il nous semble en particulier qu'elles peuvent assez facilement donner lieu à une interprétation de question rhétorique, comme en (17a), et qu'une question in situ est compatible avec la négation si on a un thème de discours négatif, comme en (17b). Le rôle des détachements (droit et/ou gauche) semble alors essentiel. Donc, selon nous, il ne faut pas chercher à expliquer (15) par une contrainte syntaxique (comme le proposent les auteurs qui parlent d'effets d'intervention $\left.{ }^{6}\right)$ mais plutôt par des contraintes discursives.

a. $\quad \mathrm{Tu}$ as vu ce que devient Jean. Il se laisse aller, ne se rase plus, s'habille mal, parle grossièrement. Dans ces conditions, il peut plaire à qui ?

b. J'aurai du mal pour les repas avec les enfants. Ma fille déteste la viande rouge, mon fils ne mange ni poisson ni légumes. Dis-moi, ton fils, il ne mange pas quoi, lui?

\footnotetext{
${ }^{5}$ On a dit la même chose des phrases interrogatives avec mouvement à longue distance (c'est-à-dire des phrases dans lesquelles le mot interrogatif est extrait d'une proposition enchâssée, comme en (i)).

(i) Qui penses-tu que Jean a vu?

(ii) a. * Penses-tu que Jean a vu qui?

b. Tu penses que Jean a vu qui?

Faute de place, nous ne considérerons pas ce type d'exemples. Notons simplement que Cheng et Rooryck en particulier jugent agrammaticaux les exemples du type de (ii)b, et les font précéder d'une étoile. Il nous semble que cela est très discutable.

${ }^{6}$ Le seul cas que l'on puisse peut-être analyser en termes d'intervention est celui où interagissent quantification, négation et question in situ. Là, seule l'interprétation écho semble disponible.

(i) Personne n'admire qui?
} 
Enfin, l'analyse des questions en qui pose problème, quand qui est sujet. Rien ne permet a priori de décider si en (18) on a une phrase interrogative avec déplacement de qui, ou une question avec qui in situ.

\section{(18) Qui vient?}

Plusieurs hypothèses syntaxiques ont été élaborées pour rendre compte de la diversité des formes du français. La question centrale porte sur le caractère optionnel ou non du mouvement. Si le mouvement est optionnel, pourquoi tous les mots interrogatifs ne peuvent-ils pas rester in situ? Et si le mouvement n'est pas optionnel, quelles sont les contraintes qui pèsent sur l'emploi de telle ou telle forme ? Plusieurs auteurs pensent qu'il existe un lien entre la position syntaxique et un type d'interprétation, et posent une parenté interprétative entre les questions clivées et les questions in situ, liée à l'existence de l'événement exprimé qui serait fortement présupposée. Est-ce exact ? Et qu'en est-il alors de la sémantique des autres formes et notamment des formes en est-ce que?

Afin de répondre à toutes ces questions, nous allons regarder dans un premier temps comment analyser les questions d'un point de vue sémantique.

\section{b) Analyse sémantique}

Depuis les travaux de Stalnaker, Kamp et Heim notamment, on distingue les approches statiques des approches dynamiques en sémantique. Du point de vue statique, une assertion dénote une proposition qui est assimilée à sa valeur de vérité, mais du point de vue dynamique, une assertion est vue comme un potentiel de changement de contexte. C'est une fonction qui transforme un contexte $\mathrm{c}$ en un contexte c'. Cela a d'importantes conséquences sur l'analyse du langage naturel : les approches dynamiques prennent en considération le discours, et peuvent donc rendre compte de phénomènes qui sortent des limites de la phrase, comme la portée des connecteurs ou le traitement des anaphores.

Pour ce qui est des questions, on peut aussi opposer les approches statiques, qui identifient le sens d'une question à l'ensemble de ses réponses (Karttunen (1977), Groenendijk \& Stokhof (1984)) et les approches plus dynamiques, comme la sémantique structurée, élaborée par van Stechow. Krifka (2001) a proposé une analyse des questions en sémantique structurée. Nous allons voir comment étendre son travail pour rendre compte du sens des diverses formes questionnantes qui ont retenu notre attention.

L'idée de la sémantique structurée est d'associer à tout énoncé assertif non pas une proposition, mais une proposition structurée sur le modèle de la paire <question, réponse $>$, de manière à rendre compte de l'articulation entre fond et focus. Une proposition est structurée, au sens où elle n'est pas assimilée à une valeur de vérité, mais est vue comme le résultat d'un calcul, le résultat de l'application d'une fonction (la question) à une valeur (la réponse). Ainsi, la phrase (20) sera associée à deux propositions structurées différentes, selon qu'elle est envisagée comme la réponse à la question (21a) ou (21b). Les formes logiques associées à (20) sont données sous (22). On voit qu'en (22) c et d l'application de la question à la réponse donne bien la proposition associée (20). 
Marie a lu La recherche du temps perdu

(21) Q. Qui a lu La recherche du temps perdu?

b. Qu'est-ce qui s'est passé ?

$$
\begin{array}{ll}
\text { a. } & <\lambda \mathrm{x}[\operatorname{read}(\mathrm{RTP})(\mathrm{x})], \mathrm{m}>7 \\
\text { b. } & <\lambda \mathrm{p}[\mathrm{p}], \operatorname{read}(\mathrm{RTP})(\mathrm{m})> \\
\text { c. } & \lambda \mathrm{x}[\operatorname{read}(\mathrm{RTP})(\mathrm{x})](\mathrm{m})=\operatorname{read}(\mathrm{RTP})(\mathrm{m}) \\
\text { d. } & \lambda \mathrm{p}[\mathrm{p}](\operatorname{read}(\mathrm{RTP})(\mathrm{m}))=\operatorname{read}(\mathrm{RTP})(\mathrm{m})
\end{array}
$$

Dans le cas des questions, on associe à la phrase la paire < background, restriction>. Cela vient de l'observation qu'une question donne souvent une information sur la nature de la réponse attendue. Qui en français sous-catégorise une réponse portant le trait humain, par opposition à que, qui demande une recherche sur les non-humains ${ }^{8}$.

$$
\begin{array}{lll}
\text { a. Qui est-ce que Marie aime? } & <\lambda \mathrm{x}[\operatorname{love}(\mathrm{x})(\mathrm{m})], \text {, humain }> \\
\text { b. Qu'est-ce que Marie aime? } & <\lambda \mathrm{x}[\operatorname{love}(\mathrm{x})(\mathrm{m})], \text {-humain }>
\end{array}
$$

Dans le cas des questions totales ou polaires, qui appellent une réponse en oui ou non, la restriction est donnée: c'est l'ensemble \{oui, non\}. Oui et non sont vus comme des opérateurs fonctionnels. Comme toute autre réponse, ils sont arguments de la question et fournissent le contenu sémantique de la réponse (cf. (25)). On donne en (26) la sémantique structurée associée à une question polaire.

(24) A a. Est-ce que Marie a lu La recherche du temps perdu?

b. $\quad \lambda f[f(\operatorname{read}(\mathrm{RTP})(\mathrm{m}))]$

$$
\mathrm{B} \text { a. Non a'. Oui }
$$
b. $\lambda \mathrm{p}[\sim \mathrm{p}]$
b'. $\lambda \mathrm{p}[\mathrm{p}]$
a. $\quad \lambda \mathrm{f}[\mathrm{f}(\operatorname{read}(\mathrm{RTP})(\mathrm{m}))](\lambda \mathrm{p}[\sim \mathrm{p}])=\sim \operatorname{Read}(\mathrm{RTP})(\mathrm{m})$
b. $\quad \lambda \mathrm{f}[\mathrm{f}(\operatorname{read}(\mathrm{RTP})(\mathrm{m}))](\lambda \mathrm{p}[\mathrm{p}])=\operatorname{Read}(\mathrm{RTP})(\mathrm{m})$
a. Est-ce que Marie a lu La recherche du temps perdu ?
b. $\quad<\lambda \mathrm{f}[\mathrm{f}(\operatorname{read}(\mathrm{RTP})(\mathrm{M}))],\{\lambda \mathrm{p}[\mathrm{p}], \lambda \mathrm{p}[\sim \mathrm{p}]\}>$

\footnotetext{
${ }^{7}$ On adopte la convention de notation suivante $: \lambda$ variable [corps de la fonction] (argument de la fonction).

${ }^{8}$ Cette caractérisation est sans doute un peu rapide. Il y a une dyssymétrie entre qui, marqué pour le trait [+ humain] et quoi, qui est non marqué plutôt que marqué pour le trait [- humain]. Qui plus est, il n'est pas toujours aisé de déterminer le trait pertinent dans l'interrogation : ainsi dans le classique 'Elle attends quoi, une fille ou un garçon?', il nous semble que les groupes nominaux 'une fille' et 'un garçon' ne référent pas à des individus, mais à des propriétés. Ce n'est peut-être pas directement l'opposition [+humain] / [-humain] qui est pertinente pour caractériser l'opposition qui / que, mais une opposition touchant au type du groupe nominal (dénotant une entité, une proprieté, un événement, une espèce...).
} 
Ce cadre présente de nombreux avantages. Il est adéquat pour rendre compte des réponses elliptiques. Il permet aussi de capter la différence entre une question et sa négation. Si l'ensemble de réponses est le même, la question posée n'est pas la même.

$$
\begin{array}{ll}
\text { a. } & \text { Est-ce que Marie est malade? } \\
\text { a'. } & <\lambda \mathrm{f}[\mathrm{f}(\mathrm{ill}(\mathrm{m}))],\{\lambda \mathrm{p}[\mathrm{p}], \lambda \mathrm{p}[\sim \mathrm{p}]\}> \\
\text { b. } & \text { Est-ce que Marie n'est pas malade? } \\
\text { b'. } & <\lambda \mathrm{f}[\mathrm{f}(\sim \text { ill }(\mathrm{m}))],\{\lambda \mathrm{p}[\mathrm{p}], \lambda \mathrm{p}[\sim \mathrm{p}]\}>
\end{array}
$$

Il rend compte de la différence entre question polaire et question alternative, qui n'attendent pas les mêmes réponses. On ne peut répondre ni oui ni non à une question alternative. C'est ce que captent les formes logiques en (28).

$$
\begin{array}{ll}
\text { a. } & \text { Marie est-elle malade? } \\
\text { a'. } & <\lambda \mathrm{f}[\mathrm{f}(\text { ill }(\mathrm{m}))],\{\lambda \mathrm{p}[\mathrm{p}], \lambda \mathrm{p}[\sim \mathrm{p}]\}> \\
\text { b. } & \text { Marie est-elle malade ou non ? } \\
\text { b'. } & <\lambda \mathrm{p} \mathrm{p},\{[\text { ill }(\mathrm{m})],[\sim \text { ill }(\mathrm{m})]\}>
\end{array}
$$

Enfin, la sémantique structurée permet de rendre compte de la notion de granularité au travers de l'idée de domaine de restriction. (29)b et b' répondent bien à (29a), mais l'une est sans doute plus appropriée que l'autre en contexte. La forme logique associée à la question elle-même peut indiquer la granularité de la réponse attendue (cf. (30)).

$$
\begin{aligned}
& \text { a. Où se trouve Paris? } \\
& \text { b. En Europe } \\
& \text { b'. En France. } \\
& \text { a. } \quad<\lambda \mathrm{x}[\text { in (Paris, } \mathrm{x})], \text { continent }> \\
& \text { b. } \quad<\lambda \mathrm{x}[\text { in (Paris, } \mathrm{x})], \text { pays }>
\end{aligned}
$$

On a aussi un cadre tout à fait adéquat pour rendre compte de l'idée de congruence des réponses : une réponse est congruente si le background de la question et celui de la réponse sont identiques et si le focus de la réponse appartient à la restriction de la question.

(31) Critère de congruence pour la paire question-réponse Q-A

$$
\begin{aligned}
& \text { Si }[[\mathrm{Q}]]=<\mathrm{B}, \mathrm{R}>\text { et }[[\mathrm{A}]]=<\mathrm{B}^{\prime}, \mathrm{F}>\text {, alors } \\
& \mathrm{B}=\mathrm{B}^{\prime} \text { et } \mathrm{F} \in \mathrm{R}
\end{aligned}
$$

Et on peut élaborer une typologie des réponses : il existe toute une palette de possibilités de réactions possibles à une question, incluant les réponses congruentes, les réponses sous ou sur informatives, les réponses inadéquates et les répliques.

$$
\text { Qu'est-ce que Marie a lu? }
$$

a. Elle a lu mon roman.

b. Elle a lu et aimé mon roman. sur-informative 


$$
\begin{array}{lll}
\text { c. Je ne sais pas. } & \text { Réplique } \\
\text { d. Jean a lu un roman . } & \text { réponse inadéquate ou implicative }
\end{array}
$$

Nous reprenons à la sémantique structurée l'idée que le sens d'une question est une fonction. Mais pour rendre compte des différences qui nous intéressent, nous avons aussi besoin de la notion de thème de discours, évoquée dans la première partie. Nous allons voir comment caractériser les conditions de légitimation de telles ou telles types de question en articulant la sémantique structurée à un cadre dialogique.

\section{La structure de l'information dans les questions : quelques contrastes}

\subsection{Les questions biaisées}

\section{Les questions déclaratives}

On dit souvent qu'en français l'intonation est porteuse de sens et permet notamment de transformer une assertion en question : le contour final montant, noté $\uparrow$, serait questionnant. On pourrait systématiquement rapprocher une phrase interrogative en est-ce que de la déclarative correspondante, assortie d'un contour final montant.

$$
\begin{aligned}
& \text { a. } \quad \text { Vous sortez } \uparrow \\
& \text { b. Est-ce que vous sortez ? }
\end{aligned}
$$

Force est de constater cependant que l'équivalence entre (33) a et b n'est pas totale. En effet, l'intonation montante par exemple, à la différence du morphème est-ce que, ne suffit pas à légitimer les items à polarité négative ${ }^{10}$.
a. Pierre a-t-il jamais compris Marie ?
a'. ?? Pierre a jamais compris Marie ?
b. Pierre a-t-il le moindre soupçon à propos de Marie ?
b'. ?? Pierre a le moindre soupçon à propos de Marie ?
c. Pierre a-t-il levé le petit doigt pour aider Marie?
c'. ?? Pierre a levé le petit doigt pour aider Marie ?

Par ailleurs, on voit que les phrases interrogatives et les déclaratives montantes ne sont pas substituables dans tous les contextes. Alors que la déclarative montante convient aux demandes de confirmation, ce n'est pas le cas des interrogatives, qui sont incompatibles avec n'est-ce pas.

\footnotetext{
${ }^{9}$ Cf. Büring et la notion de topique implicatif, marqué par un accent spécifique et obligatoire en anglais et en allemand.

${ }^{10}$ Un item à polarité négative (IPN) est une expression lexicale ou syntagmatique qui ne peut pas apparaître dans une phrase déclarative affirmative, mais apparait en revanche dans les phrases interrogatives ou négatives. Les IPN en (34) sont jamais, qui a le sens de un jour, le moindre et l'expression lever le petit doigt.
} 


\author{
a. Vous sortez, n'est-ce pas $\uparrow$ \\ b. * Est-ce que vous sortez, n'est-ce pas ?
}

C'est donc que les énoncés déclaratifs avec contour montant sont différents des phrases interrogatives et en un sens ne constituent pas de vraies questions. Les déclaratives montantes ressemblent à des questions mais n'ont pas toutes les propriétés des questions. Il est facile de capter ce fait et de prédire le biais associé aux déclaratives montantes dans notre modèle. En effet, l'impact d'un énoncé sur le contexte conversationnel n'est pas le même dans le cas des phrases déclaratives et interrogatives. L'emploi d'une phrase interrogative q entraîne l'ajout de q dans QUD, mais ne change rien à FACTS. En revanche, l'impact d'une déclarative $\mathrm{p}$ sur le contexte est double : il y a mise à jour de QUD (augmenté de ?p) et mise à jour de FACTS (auquel on ajoute p). C'est cet ajout de p dans FACTS qui explique le biais associé aux déclaratives montantes ${ }^{11}$.

\title{
Les interro-négatives
}

Un second fait est connu : les phrases interro-négatives comme (36b) appellent une réponse positive. Là encore, on parle de biais. On pourrait dire que les phrases interro-négatives sont des demandes d'infirmation.

$$
\begin{array}{ll}
\text { a. } & \text { Jean est-il communiste ? } \\
\text { b. } & \text { Jean n'est-il pas communiste ? } \\
\text { a. } & <\lambda \mathrm{f}[\mathrm{f}(\operatorname{communiste}(\mathrm{j}))],\{\lambda \mathrm{p}[\mathrm{p}], \lambda \mathrm{p}[\sim \mathrm{p}]\} \\
\text { b. } & <\lambda \mathrm{f}[\mathrm{f}(\sim \text { communiste }(\mathrm{j}))],\{\lambda \mathrm{p}[\mathrm{p}], \lambda \mathrm{p}[\sim \mathrm{p}]\}
\end{array}
$$

Dans notre modèle, (36)a et b n'ont pas le même sens (cf (37)) et l'on peut expliquer pourquoi ces deux phrases n'ont pas les mêmes conditions d'emploi. Pourquoi choisir de poser une question négative plutôt qu'une question positive ? Si le locuteur n'a pas d'idée sur $\mathrm{p}$, choisir l'interro-négative ? p plutôt que ?p serait contraire au principe d'économie générale qui règle l'usage du langage. Si le locuteur a des raisons de croire p, il peut se trouver dans deux situations : ou bien, il demande à son interlocuteur de confirmer cette croyance, et dans ce cas la déclarative questionnante est tout à fait appropriée ; ou bien le locuteur sait déjà que p et souhaite activer cette connaissance chez son interlocuteur. C'est dans ce cas-là que la phrase interro-négative est appropriée. Ce qui distingue la demande de confirmation de la demande d'infirmation, c'est que dans le second cas, le locuteur sait que p, et fait un

\footnotetext{
${ }^{11}$ Ce n'est pas le contour montant en tant que tel qui transforme l'assertion en question. Plusieurs études comme celles de Beun (1988) ou de Gustafson-Capková (2005) ont montré que bien des phrases déclaratives sont interprétées comme des questions, alors même qu'elles ne sont pas associées à un contour final montant. Notamment dans les interactions de services, où le rôle de chaque interlocuteur est fortement conventionalisé. A titre d'exemple, on a enregistré des échanges comme le suivant :

Guichetier à la SNCF: $\quad$ - Vous voulezpartir le plus rapidement possible? (B)

Client - Oui. Dès que possible.
} 
usage rhétorique de la question. D'où le biais. Une observation conforte cette hypothèse : on ne peut pas faire précéder une question négative de «Je n'ai pas la moindre idée là-dessus. Dis-moi...»

a. Je n'ai pas la moindre idée là-dessus. Dis-moi, Jean est-il communiste ?

b. \# Je n'ai pas la moindre idée là-dessus. Dis-moi, Jean n'est-il pas communiste?

Dans notre cadre, on rend compte de cette donnée par une contrainte discursive qui règle la mise à jour de QUD : on ne peut introduire un élément négatif dans QUD que si la proposition positive correspondante se trouve déjà dans FACTS. Cette contrainte est générale. Dans le cas de (36b), elle amène l'interlocuteur à accommoder le fait que Jean est communiste dans FACTS.

\subsection{Questions in situ}

Qu'est-ce qui distingue, en français, les questions antéposées des questions in situ ? Selon Chang (1997), Cheng \& Rooryck (2000) entres autres, il existe une parenté interprétative entre les questions in situ (39a) et les questions clivées (39c). Toutes deux seraient utilisées dans des contextes où l'existence de l'événement exprimé est fortement présupposée, à la différence des questions antéposées (39d) et renforcées (39e). (39a) serait appropriée uniquement dans un contexte où l'on présuppose que Marie a acheté quelque chose. Cheng \& Rooryck ajoutent à leur étude des considérations prosodiques : selon eux, à la différence des questions antéposées dont l'intonation peut être soit montante soit descendante, les questions in situ sont toujours montantes, comme les questions totales, qu'elles soient déclaratives ou interrogatives (cf. $39 \mathrm{~b})^{12}$.
a. Marie a acheté quoi ?
intonation montante
b. (Est-ce que) Marie a acheté un livre ? intonation montante
c. C'est quoi que Marie a acheté ?
d. Qu'a acheté Marie ?
intonation wh
e. Qu'est-ce que Marie a acheté ?
intonation wh

Les données présentées ci-dessus nous semblent tout à fait discutables. D'une part, des études récentes (Bartels (1999), Beyssade \& al. (2004)) ont été menées sur le sens de l'intonation, à la fois en anglais et en français, et ont montré que la prosodie de phrases comme (39) a et b n'est pas nécessairement montante. D'autre part, l'idée même d'une parenté interprétative entre (39) a et c a été remise en cause par Obenauer (1994). Nous pensons comme Obenauer qu'à la différence des questions clivées, les questions in situ ne portent pas de présupposition d'existence. Preuve en est le contraste (40), qui montre bien que si la clivée exclut une réponse négative du type rien ou personne, ce n'est pas le cas de la question in situ.

\footnotetext{
12 Selon Cheng et Rooryck, le mot interrogatif in situ en français est légitimé par un morphème [Q] sous-spécifié, qui est désambiguiisé en [Q : wh] par le mouvement des traits. Il en résulte l'intonation caractéristique de cette construction.
} 
a. Tu as vu qui, quelqu'un ou personne?

a'. ?? C'est qui que tu as vu, quelqu'un ou personne?

b. A la manif, tu as retrouvé qui, des copains ou personne ?

b'. ?? A la manif, c'est qui que tu as retrouvé, des copains ou personne?

On peut aussi ajouter l'observation déjà présente dans Mathieu (2004) selon laquelle on peut bien répondre à une question in situ par rien ou personne. Enfin, si les questions in situ portaient une présupposition d'existence, (42) devrait être mal formé.

(41) A: Tu fais quoi, toi, pendant les vacances?

B: Rien.

a. Tu veux quoi, du thé, du café ou rien?

Cela ne veut pas dire qu'il n'y ait aucune différence entre question in situ et question antéposée. Mais selon nous, la différence concerne l'impact de la phrase sur le thème de discours. Les questions antéposées peuvent introduire un nouveau thème de discours, ce qui n'est pas le cas des questions in situ, qui continuent sur le thème de discours en cours. Dans notre modèle, les questions in situ sont analysées comme des phrases déclaratives, donc elles sont soumises aux mêmes contraintes d'emploi que les phrases déclaratives. Or il en est une qui stipule qu'une phrase déclarative n'est appropriée que si elle élabore le thème de discours en $\operatorname{cours}^{13}$. Un petit fait corrobore cette analyse. On trouve peu de titres de roman, de journaux ou d'articles scientifiques qui prennent la forme d'une question, mais quand ils sont interrogatifs, c'est en général la forme antéposée qui est choisie (Où va la France?, A quoi rêvent les jeunes filles ?), et jamais ou presque jamais la forme in situ (\# La France va où ?, \# Les jeunes filles rêvent à quoi ?). C'est sans doute parce que le titre a pour fonction d'introduire un nouveau thème de discours et qu'une question in situ ne peut que continuer sur le thème de discours en cours.

\subsection{L'accentuation des questions}

Il existe des travaux en anglais qui portent sur l'interprétation des accents dans les questions avec mot wh. Lambrecht \& Michaelis montrent par exemple que les questions (43) a et b n'ont pas le même sens, ni les mêmes conditions d'emploi.

$$
\begin{aligned}
& \text { a. So WHEN are we going to Edinburgh? } \\
& \text { b. So, when are we going to EDINBURGH? }
\end{aligned}
$$

En (43a), le mot wh est accentué et la question signifie C'est quand qu'on va à Edinbourg ? Le fait d'aller à Edinbourg est acquis, présupposé, la question ne porte que sur le moment où l'on y va. Une autre paraphrase serait On va à Edinbourg, mais quand?

\footnotetext{
${ }^{13}$ Une phrase déclarative peut changer le thème de discours, mais elle est alors associée à une prosodie spécifique (cf. analyse de l'accent $\mathrm{B}$ par Büring). Par ailleurs, même dans ce cas, le thème de discours introduit est en relation avec le thème de discours précédent. Il ne peut pas être complètement indépendant du thème de discours précédent (voir aussi la notion de discourse-pop en SDRT).
} 
En revanche, en (43b), on a en fait deux questions, qui dépendent l'une de l'autre. (43b) signifie Si on va à Edinbourg, quand y va-t-on?, ou Est-ce qu'on va à Edinbourg et quand?

Engdahl (2006) fait une description analogue de l'accentuation dans les questions totales en anglais. Elle compare (44) a et b et glose (44b) par une question clivée Est-ce que c'est John qui a lu le journal?
a. Did John read the newspaper?
b. Did JOHN read the newspaper?

En français, on observe quelque chose de comparable. Prenons une phrase interrogative comme (45). Cette phrase peut donner lieu à plusieurs réalisations prosodiques, qui se distinguent par la place du ton bas dans la séquence. Des études sur corpus nous ont permis de constater que ce ton bas pouvait s'ancrer soit sur le mot interrogatif, soit sur le clitique $t u$, soit sur le participe passé caché, soit en fin d'énoncé, d'où quatre découpages prosodiques différents, donnés respectivement sous (46).

Où as-tu caché ton sac?

$$
\begin{array}{ll}
\text { a. } & \text { (Où) (as-tu caché ton sac) } \\
\text { b. } & \text { (Où as-tu) (caché ton sac) } \\
\text { c. } & \text { (Où as-tu caché) (ton sac) } \\
\text { d. } & \text { (Où as-tu caché ton sac) }
\end{array}
$$

Il nous semble que ces réalisations prosodiques ne véhiculent pas exactement le même sens. Notre hypothèse est qu'on peut maximiser la ressemblance entre l'analyse des phrases déclaratives et celle des phrases interrogatives, et considérer que ce ton bas marque la frontière gauche du domaine focal. Il marquerait dans les assertions la frontière gauche du constituant spécifiquement asserté et dans les questions la frontière gauche du constituant spécifiquement interrogé. Conséquence : dans les questions, le domaine focal ne se réduit pas nécessairement au seul mot interrogatif. Dans le cas où le domaine focal inclut le mot interrogatif mais le dépasse, on aurait en quelque sorte deux questions en une, ou une question sous condition. (46) a-c correspondraient respectivement aux paraphrases suivantes :

$$
\begin{aligned}
& \text { a. Où as-tu caché ton sac ? } \\
& \text { b. Si c'est toi qui as caché ton sac, où l'as-tu caché ? } \\
& \text { b'. Est-ce toi qui as caché ton sac, et où ? } \\
& \text { c. Si tu as caché ton sac, où l'as-tu caché ? } \\
& \text { c'. As-tu caché ton sac, et où ? }
\end{aligned}
$$

Cette analyse, qui demande à être confortée empiriquement, a quelques implications importantes. En particulier, elle permet d'importer la distinction entre focus large et étroit des assertions aux questions. On aurait des questions all focus et des questions narrow focus comme on a des assertions all focus et des assertions narrow focus, 
et ce aussi bien pour les interrogatives polaires que pour les interrogatives partielles (avec mot interrogatif). Cette analyse remet donc en cause l'idée répandue selon laquelle dans les questions, le mot interrogatif et le focus se confondent.

\section{Conclusion}

S'il existe une importante littérature sur la syntaxe des questions, notamment dans le cadre générativiste, il existe en revanche peu d'études systématiques de la sémantique et de la pragmatique des questions. C'est sans doute qu'une telle entreprise est d'autant plus difficile qu'elle requiert un travail d'analyse des contextes: si on cherche à corréler formes et contextes d'emploi - comme on l'a fait ici sur quelques exemples - il faut établir une typologie des contextes, savoir isoler les paramètres pertinents pour caractériser un contexte, le comparer avec un autre...

Même si les résultats présentés ici sont très partiels, ils montrent l'importance de la structure de l'information dans les questions, et nous ont conduit à distinguer au moins trois dimensions dans le contexte : le fond, le focus et le topique de discours. On peut y voir un premier pas dans l'élaboration d'une typologie des questions, mettant en avant ce qui les distingue en termes de dénotation, en termes d'implicatures (une question présuppose-t-elle certaines connaissances, la réponse attendue est-elle orientée, doit-elle être exhaustive... ?), et en termes dialogiques (la question ouvre-t-elle ou non un nouveau segment discursif, est-elle rhétorique, polémique... ?). Nous aimerions conclure en disant que si le sens d'une assertion, c'est un potentiel de changement de contexte, le sens d'une question, c'est un potentiel de changement de topique de discours. 
Claire Beyssade

\section{RÉFÉRENCES}

BARTELS, C., 1999, Towards a Compositional Interpretation of English Statement and Question Intonation. New-York \& London : Garland.

BEun, R.-J., 1990, «The Recognition of Dutch Declarative Questions ». Journal of Pragmatics 14 : 39-56.

Beyssade C., E. Delais, J.-M. Marandin, A. Rialland, 2004, "Prosody and Information », in H. de SwART and F. CORBLIN (eds), Handbook of French Semantics, Stanford : CSLI.

Beyssade, C. ; E. Delais, J.-M. Marandin, A. Rialland et M. De Fornel, 2004, « Le sens des contours intonatifs en français : croyances compatibles ou conflictuelles ? ", Actes de JEP-TALN 2004.

Beyssade, C., J.-M. MARAndin \& A. Rialland, 2003, « Ground/focus revisited. A perspective from French », Proceedings of LSRL 2001, Benjamins.

BurIng, D., 1997, The Meaning of Topic and Focus: The 59th Street Bridge Accent. London : Routledge.

CHANG, L., 1997, «Wh-In-Situ Phenomena in French ». Thèse de doctorat University of British Colombia.

Cheng, L., J. RoOryCK, 2000, « Licensing Wh-In-Situ ». Syntax 3 :1, 1-19.

Culioli, A., 1990, «Donc», Pour une linguistique de l'énonciation, Tome 1, Ophrys.

Delais-Roussarie, E., 2005, Phonologie et Grammaire : études et modélisation des interfaces prosodiques. HDR, Université de Toulouse-le Mirail.

Di CRISTO, A., 1999, «Le cadre accentuel du français contemporain ». Première partie. Langues 3 (2): 184-205. Deuxième partie. Langues 4 (2): 258-267.

ENGDAHL, E., 2006, « Information packaging in question », Proceedings of CSSP 2005.

ENGDAHL, E., E. VALLDUVI, 1994, «Information packaging and grammar architecture : a constraint-based approach", in E. ENGDAHL (ed.) Integrating information structure into constraint-based and categorial approaches, DYANA-2 Report.

GinzBurg, J., 1997, «On some Semantic Consequences of Turn Taking », in P. DEKKER et al. (eds.), Proceedings of the 11th Amsterdam Colloquium on Formal Semantics and Logic, 145-150, Amsterdam: ILLC.

GinzBurg, J., à paraittre, A Semantics for Interaction in Dialogue, CSLI Publications and University of Chicago Press.

GroenendijK, J., M. Stokhof, 1984, Studies on the Semantics of Questions and the Pragmatics of Answers, Ph.D. dissertation, Amsterdam.

Gustafson-CapkovÁ, S., 2005, Integrating Prosody in an Account of Discourse Structure, Thesis, Stockholm University.

HallidaY, M., 1967, « Notes on transitivity and theme in English, part II », Journal of linguistics 3 : 199-244. 
JACKENDOFF, R., 1972, Semantic interpretation in Generative Grammar. Cambridge (Ma.). MIT Press.

KartTunen, L., 1977, «Syntax and semantics of questions », Linguistics and Philosophy, 1: 3-44.

KrIFKA, M., 2001, «For a Structured Meaning Account of Questions and Answers », in C. FERY et al. (eds.), Audiatur Vox Sapientia. A Festschrift for Arnim von Stechow. Berlin : Akademie Verlag. 287-319.

LAMBRECHT, K., 1994, Information structure and sentence form, Cambridge : Cambridge university Press.

LAMBRECHT, K., L. Michaelis, 1998, «Sentence accent in information question : default and projection », Linguistics and Philosophy, 21: 477-544.

MARANDIN, J.-M., 2003, «Inversion du sujet et structure de l'information dans les langues romanes ", in D. GoDARD (ed), Langues romanes. Problèmes de la phrase simple, Paris: Éditions du CNRS.

MARANDin, J.-M., 2005, «Formatage de l'information: focus et contexte», in F. Corblin, C. GARDENT (eds), Interpréter en contexte, 31-80, Paris, Hermès.

Mathieu, E., 2004, "The mapping of form and interpretation: the case of optional wh-movement in French », Lingua 114 : 1090-1132.

Obenauer, H.-G., 1994, Aspects de la Syntaxe A-Barre, Effets d'Intervention et Mowvements des Quantifieurs. Thèse de Doctorat d'Etat Université Paris VIII.

OBENAUER, H.-G., 2005, La syntaxe des questions non standard: les questions de surprise désapprobation en Bellunese, Recherches Linguistiques de Vincennes, 33 : 55-90.

Paul, H., 1880. Prinzipien der Sprachgeschichte, Niemeyer.

Steedman, M., 2000, The syntactic interface, Cambridge : MIT Press.

VALLDUVÍ, E., 1992, The informational component, New-York : Garland. 
